\title{
SABER LEGO Y EXPERTO EN SALUD
}

\author{
KNOW LEGO AND EXPERT IN HEALTH
}

\section{CONHECIMENTO LEIGO E ESPECIALIZADO EM SAÚDE}

\author{
Rodolfo Levin Echeverri ${ }^{1}$
}

\begin{abstract}
Resumen: En el presente artículo se proponen tres insumos orientados a la reflexión teórico metodológica sobre la pertinencia de estrategias de investigación cualitativa para comprender la relevancia del conocimiento lego en salud y su importancia en la gestión de un sistema sanitario : 1 La circulación de saberes legos y expertos en torno a la enfermedad renal crónica, 2- Proceso de adquisición de saberes expertos por parte de una comunidad de legos ante la organización de un servicio de salud, 3-Los vínculos entre el conocimiento lego y el conocimiento médico en tiempos de internet e incorporación normativa de la figura del paciente experto en un sistema de salud. El objetivo de este artículo es sistematizar hallazgos empíricos y convergencias teóricas sobre la utilidad de la estrategia cualitativa en la investigación social en salud y observar nexos probables con metodologías ancladas en la investigación participativa en salud.
\end{abstract}

Palabras clave: Investigación cualitativa; Conocimiento lego y experto; Participación social.

Abstract: This article proposes three inputs aimed at theoretical methodological reflection on the relevance of qualitative research strategies to understand the relevance of lay knowledge in health and its importance in the management of a health system: 1 The circulation of lay knowledge and experts around chronic kidney disease, 2- Process of acquiring expert knowledge by a community of lay people before the organization of a health service, 3-The links between lay knowledge and medical knowledge in times of internet and incorporation normative of the figure of the expert patient in a health system. The objective of this article is to systematize empirical findings and theoretical convergences about the usefulness of the qualitative strategy in social health research and to observe probable links with methodologies anchored in participatory health research.

Keywords: Qualitative research; Lay and expert knowledge; Social participation.

Resumo: Este artigo propõe três insumos destinados à reflexão metodológica teórica sobre a pertinência das estratégias de pesquisa qualitativa para compreender a relevância do conhecimento leigo em saúde e sua importância na gestão de um sistema de saúde: 1 A circulação de conhecimentos leigos e especialistas em torno da doença renal crônica, 2- Processo de aquisição de conhecimento especializado por uma comunidade de leigos diante da organização de um serviço de saúde, 3-Os vínculos entre conhecimento leigo e conhecimento médico em tempos de internet e incorporação normativa da figura do paciente especialista em um sistema de saúde. O objetivo deste artigo é sistematizar descobertas empíricas e convergências teóricas sobre a utilidade da estratégia qualitativa na investigação social em saúde e observar possíveis ligações com metodologias ancoradas na investigação participativa em saúde.

Palavras-chave: Investigação qualitativa; Conhecimento leigo e especializado; Participação social.

\footnotetext{
${ }^{1}$ Magister en. Sociologia. FCS/UDELAR.Director Instituto de Higiene.Laboratorio de Ciencias Sociales em Salud.FMED/UDELAR Montevideo. Uruguay. E-mail: rlevin@higiene.edu.uy; rodo.levin@gmail.com
} 


\section{Introducción}

Las diferentes formas de participación social en la construcción de un sistema de salud en Uruguay tienen el doble atractivo de ser, por un lado, un laboratorio de experiencias participativas en salud y por otro, un espacio para la investigación social en salud desde una perspectiva cualitativa. Desde el año 2007 se desarrolla en el país la reforma del sistema sanitario que adquirió el nombre de Sistema Nacional Integrado de Salud (SNIS), (LEY 18.211,2008). Dicha reforma se asentó en tres pilares básicos: Cambio del modelo de financiamiento, cambio del modelo de atención y cambio del modelo de gestión. En este último se encuentra la preocupación de nuestro artículo. ¿Dónde está el núcleo de la preocupación ontológica?: En la incorporación de nuevos actores sociales a los procesos de decisión en la gestión del Sistema de Salud (FUENTES,2010). Ello constituye una originalidad sustantiva en el funcionamiento del sistema a la vez de ser un espacio para la investigación social. Subsidiarias y convergentes a la preocupación ontológica, aparecen las siguientes preguntas:

- ¿Existen indicadores de empoderamiento por parte de actores socialmente subordinados en el sistema de salud?

- ¿cómo se expresa la interacción social en los diferentes procesos de decisión?

- ¿qué técnicas, desde la investigación cualitativa en salud, se revelan eficaces para construir evidencia empírica robusta respecto a los actores sociales, su trayectoria y posición en el sistema de salud?

- ¿Son eficaces las categorías analíticas de conocimiento lego y conocimiento experto en salud para comprender los diversos procesos de interacción social en el campo de la salud?

A tal efecto se proponen tres insumos para una reflexión teórico metodológica sobre la pertinencia de estrategias de investigación cualitativa y participativa en salud. A partir de dichos insumos se problematiza la pertinencia de la investigación cualitativa y participativa en salud con dos abordajes técnicos diferentes. Por una parte, la entrevista en profundidad (como fuente secundaria) y el Juicio Ciudadano o Asamblea por Consenso y por otro lado se observan los vínculos entre el conocimiento lego y el conocimiento médico en tiempos de internet y la incorporación normativa de la figura del paciente experto en un sistema de salud. 


\section{La circulación de saberes legos y expertos en torno a la enfermedad renal crónica} (Insumo 1)

\subsection{Objetivo}

Detectar la adquisición y circulación de un nuevo saber por parte de los agentes no expertos (portadores de ERC y familiares) y las formas que se expresa en la interacción social con el saber experto profesional y con el resto del contexto sociocultural donde se ha desarrollado ese nuevo conocimiento (LEVIN, 2019)

\subsection{Metodología}

Análisis sistemático de fuente secundaria. Constituida por 30 entrevistas en profundidad realizadas en Uruguay y que formaron parte de: Un estudio comparativo sobre la atención a la enfermedad renal crónica: Brasil, México y Uruguay, Investigador responsable: Dr. Francisco J. Mercado Martínez (Universidad de Guadalajara, México). Investigador asociado: Rodolfo Levin (Universidad de la República, Uruguay) 20142016 (MERCADO; LEVIN,2017). Finalizada esa etapa de la investigación se indagó por parte del investigador asociado- en la comprensión del universo de interacciones sociales entre el saber experto médico y el saber lego de los trasplantados renales. Existe una hipótesis subyacente respecto a que en dicho universo ocurren hechos sociales invisibles para los tomadores de decisión. Las políticas de salud en el área de la enfermedad renal subestiman esa dimensión peculiar: el saber y sentir del trasplantado renal. En este punto debe señalarse la utilización del corpus narrativo de 30 entrevistas en profundidad con un objetivo diferente al que les dio origen. Dicha decisión, con la anuencia del investigador principal, significó la posibilidad de multiplicar- a partir de objetivos distintos- el capital heurístico de la investigación original (LEVIN, 2019)

\subsection{Resultados principales}

En virtud del acervo que generó la investigación realizada - en este caso solo Uruguay- conformado por las entrevistas en profundidad a enfermos renales y familiares, se hizo uso de dicha evidencia empírica bajo un esquema epistemológico similar a la investigación principal: un enfoque crítico interpretativo. El criterio de búsqueda de indicios discursivos se centró en detectar la adquisición y circulación de un nuevo saber por parte de los agentes no expertos (portadores de ERC y familiares) y las formas que se 
expresa en la interacción social con el saber experto profesional. En esta ocasión-a partir de la narrativa de los hablantes- se construyeron tres dimensiones analíticas: A- LA CARRERA DEL PACIENTE: Consiste en una homología respecto al concepto de "carrera moral" de Erving Goffman (1972) identificando hitos en su desarrollo: Comienzo (irrupción de la enfermedad), Puntos culminantes (el momento del trasplante) y Resultados (la vida cotidiana), marcadas, cada una de ellas por configuraciones estables de signos y síntomas. Se optó por denominar "carrera del paciente" a la secuencia de acontecimientos sociales que conforman el proceso del ERC, donde los puntos de esa carrera suponen la asunción de roles diferentes en cuanto a la incorporación y puesta en práctica de saberes adquiridos. Las dimensiones analíticas B y C están constituidas por lo que se ha denominado CONOCIMIENTO TIPO 1 y CONOCIMIENTO TIPO 2. En el primer caso refiere al conocimiento adquirido en la "carrera de la enfermedad" que realiza el agente sobre aspectos biológicos, químicos, de diagnóstico, terapéuticos, profilácticos y sociales de la ERC. En el caso del conocimiento tipo 2 refiere al escenario político, social y asistencial que contextualiza a la ERC. Tanto para el contenido discursivo de la variable conocimiento tipo 1 como para conocimiento tipo 2 se identifica a los relatos que expresan la apropiación reflexiva del conocimiento por parte de los agentes sociales. Ello es una alusión directa a Anthony Giddens (1994) en cuanto dicho autor define la reflexividad como una cualidad específica de las prácticas sociales. Es indisociable de la recursividad, entendida esta como la reproducción ordenada de las prácticas sociales. Ambas constituyen el punto de intersección en las diferentes capacidades de los agentes: Tener noción de saber lo que hacen y porqué lo hacen, es decir, tener la capacidad de monitorear sus acciones. Esto a su vez, le permite interacción con otros agentes generando a su vez nuevas prácticas sociales autorreproducidas reflexivamente. Supone una relación dialéctica entre el saber profano y el experto. En esa relación habitan la generación e intercambio de saberes donde el conocimiento lego- desde la reflexividad del agenteincorpora un nuevo conocimiento que a su vez ingresa en el espacio de la disputa social, normalmente bajo la forma del conflicto y el acuerdo. La reflexividad, en tanto concepto teórico interpretado bajo el lente de Anthony Giddens (1994) habilita a comprender la circulación social de nuevos conocimientos por parte de los agentes (portadores de ERC y familiares) desarrollando nuevos y recursivos procesos de interacción social con el conocimiento experto. Ello se expresa, en la tríada conceptual presentada: Carrera del paciente, conocimiento Tipo 1 y conocimiento Tipo 2 (LEVIN, 2019) 


\subsection{Discusión de resultados}

Los indicadores presentados fortalecen la forma y modo en que el conocimiento no experto interactúa en el campo de la salud renal significando, eventualmente, un instrumento para evaluar la toma de decisiones orientadas a mejorar la calidad de vida de quienes son portadores de ERC y su acceso a las prestaciones de los servicios de salud. Otro elemento significativo es observar el papel heterogéneo y heterodoxo que cumplen las diversas asociaciones de usuarios. Llamativamente, en un contexto institucional que ha normatizado- y privilegiado - el rol y participación de usuarios y trabajadores en el sistema de salud no se detecta un papel de gran incidencia en la gestión del propio sistema (LEVIN, 2019)

\section{Proceso de adquisición de saberes expertos por parte de una comunidad de legos} ante las formas de organización y funcionamiento de un Hospital Universitario (Insumo 2)

Este insumo forma parte de un proceso reflexivo en relación al Proyecto Juicio Ciudadano al Hospital de Clínicas. Proyecto CSIC.Art.2. 2014-2016. El mismo fue una iniciativa desarrollada desde el Laboratorio de Ciencias Sociales en Salud (Instituto de Higiene-Facultad de Medicina-Universidad de la República) entre los meses de noviembre de 2014 y julio de 2016. El mismo fue seleccionado por la Comisión Sectorial de Investigación Científica (CSIC) en el marco de la convocatoria 2014 de los denominados llamados Art.2 (Difusión de temas científicos orientados a la comprensión pública) (LEVIN, 2019)

\subsection{Objetivos}

A: Abordar una metodología participativa con centro en el saber hacer de ciudadanos legos sobre la materia en estudio: Inserción del Hospital de Clínicas en el SNIS y las consecuentes formas en que dicho saber se construye y hace público. $B$ : Sistematizar la convergencia de saberes ciudadanos diversos y evaluar si es posible generar un nuevo saber participativo como insumo para diseño de políticas públicas (LEVIN, 2019). 


\subsection{Metodología}

El proyecto tomó el modelo de los Juicios ciudadanos o Asambleas de consenso que tuvieron su origen en Dinamarca hacia finales de los años 80 y que son definidos como un mecanismo de participación ciudadana para el tratamiento de aspectos científico-tecnológicos controversiales y de aparente complejidad para la comprensión del saber lego. El objetivo es incidir en la elaboración de las políticas públicas (LÁZARO et al., 2013). En este caso el proceso involucró a tres grandes grupos: El Grupo Coordinador (responsables del Proyecto), un Panel Ciudadano compuesto por personas "no expertas ni implicadas en la temática en cuestión" seleccionadas a través de un llamado público y un Grupo de Expertos que funcionó como un comité de asesores que orientó, a través de documentos y participación en sesiones del panel, al grupo coordinador y al propio panel de ciudadanos. En el transcurso del trabajo se realizaron múltiples actividades: reuniones pautadas del panel con grupo coordinador y asesores, reuniones de trabajo del panel, entrevistas a informantes calificados y visitas al Hospital Universitario culminando con una Audiencia Pública en junio de 2016. El Proyecto supuso la convergencia de saberes ciudadanos diversos junto a una praxis académica de disciplinas distintas: Medicina, Salud Pública, Sociología y Comunicación. Originalmente el proyecto no contempló las formalidades de un proyecto de investigación. Asume dicha condición ex post y donde, efectivamente hubo recolección sistemática y protocolizada de una evidencia empírica heterogénea y de una gran densidad informativa (LEVIN, 2019)

\subsection{Resultados principales}

Más allá de los resultados (“veredicto”) volcados en la Audiencia Pública final adquiere principal relevancia la percepción de los integrantes del Panel (agentes legos) una vez finalizado el trayecto de 6 meses de duración del Juicio Ciudadano. En razón de ello se aplicó a los integrantes del Panel Ciudadano un cuestionario de evaluación individual a partir del cual se sistematizaron las siguientes dimensiones y sus indicadores y/o percepciones. DIMENSIÓN 1: EVALUACIÓN DEL CONOCIMIENTO ADQUIRIDO EN EL TRAYECTO DEL PROYECTO cuyos indicadores son 1: el proceso cognitivo del saber lego; 2: Historia y presente de proyectos sobre el Hospital Universitario; 3: el rol de los usuarios; 4: El conflicto permanente entre actores. 5: la impugnación social sobre corporación médica; 6: "La camiseta" o el sentido de 
pertenencia institucional de actores colectivos e individuales. DIMENSIÓN 2: ROL DE LOS ASESORES expresado empíricamente en los indicadores: a: Grupos; b: Alineados /no alineados. ;c: Arrepentidos y militantes. d: Visión estratégica/visión dogmática. DIMENSIÓN 3: EL ROL COMO INTEGRANTE DEL PANEL CIUDADANO (o como transitar desde el conocimiento lego al conocimiento no lego) DIMENSIÓN 4: AUDIENCIA PÚBLICA (AP). La instancia de la AP del 23.06.2016 fue el hito fundamental del proyecto, el punto de encuentro de todos los esfuerzos del equipo organizador y del Panel Ciudadano y donde se depositaron todas las expectativas de culminación del trabajo (LEVIN, 2019)

\section{Los vínculos entre el conocimiento lego y el conocimiento médico en tiempos de internet e incorporación normativa de la figura del paciente experto en un sistema de salud (Insumo 3)}

Desde finales del siglo XX y en las dos primeras décadas del siglo XXI es insoslayable la digitalización de la vida cotidiana. Ello ha implicado todas las áreas de la actividad humana y el sector sanitario no es ajeno a ello. En términos de interacción social la relación entre el profesional médico y el paciente está fuertemente impactada por el citado fenómeno, sobre todo en lo referido a la información e intercambio entre ambos actores, relación históricamente asimétrica en razón de diferentes posiciones de saber y autoridad social. En tal sentido- y convergente con artículo presentado en CIAIQ 2019hemos tomado dos revisiones bibliográficas referidas, en primer lugar, a la relación entre el conocimiento lego y experto en salud en tiempos de internet (GARBIN; PEREIRA NETO; GUILAM, 2008) y en segundo lugar la incorporación normativa de la figura del paciente experto en un sistema de salud (GREENER, 2008).

\subsection{Los vínculos entre el conocimiento lego y el conocimiento médico en tiempos de internet}

En el caso citado, luego de una vasta revisión bibliográfica, los autores proponen que los pacientes que tienen un adecuado acceso a informaciones a través de internet transitan un proceso de empoderamiento social y esta nueva condición puede transformar la relación entre el médico y su paciente. Ello aparece como un fenómeno social complejo y desde el propio artículo se advierten distintas posiciones. En primer lugar, se alude al hecho que la adquisición de nuevos saberes por parte de los pacientes a través de internet cuestiona el estatus y la autoridad de la profesión médica, contribuyendo a su 
desprofesionalización. En tal sentido aluden a publicaciones de Marie Haug y Elliot Friedson donde plantean perspectivas diferentes. A principios de los 70 Haug sugiere que la elevación del nivel educacional de las poblaciones y el acceso a un mejor conocimiento técnico habría de promover, por parte de las ocupaciones profesionales, la pérdida de los monopolios del conocimiento (GARBIN; PEREIRA NETO; GUILAM, 2008) Esta aseveración parece más pertinente aún luego de la irrupción de internet y particularmente en el sector de la salud. Sin embargo, Elliot Friedson, a finales de los años 80 e inicio de los 90, y ya en el campo especifico de la relación médico - paciente entiende que la misma se basa en la autoridad del primero en relación con el segundo. Esta autoridad deriva del conocimiento que tiene el primero sobre el problema que vive el segundo. A medida que este conocimiento se aplica y resuelve, en general, los problemas del paciente se someten a esta autoridad. Para él, la distancia entre el conocimiento lego y el científico sobre un tema dado sigue existiendo (GARBIN; PEREIRA NETO; GUILAM,2008).

\begin{abstract}
Aunque los estudios de Freidson y Haug preceden al auge de Internet, podemos deducir que Freidson defendería la posición opuesta a la que tenía Haug con respecto al poder del paciente experto para cambiar la relación médicopaciente. Para Freidson, el acceso a la información no reduce la autoridad del médico y el prestigio social, ni promueve su desprofesionalización. Por el contrario, al buscar información sobre la ciencia, la gente común se está volviendo cada vez más parte de la lógica dominante de la medicina occidental. Con esto, el paciente siembra el terreno para que el diagnóstico y el tratamiento médico sean más fácilmente aceptados. El profesional, por otro lado, no puede estar desactualizado. Debe ser más experto que su paciente (GARBIN; PEREIRA NETO; GUILAM, 2008, p. 581).
\end{abstract}

La mayoría de los artículos relevados entienden que los pacientes que tienen acceso a la información a través de Internet, se vuelven potencialmente poderosos (empoderamiento social ).La relevancia del artículo citado estriba en sistematizar tres posiciones distintas en cuanto a la interacción entre el conocimiento experto y lego: 1 : El empoderamiento social del agente lego socava estado y autoridad de la profesión médica; 2: Existe el empoderamiento social del agente lego pero genera un fortalecimiento de la racionalidad biomédica dado que no existe un reemplazo de un conocimiento por otro ; 3: Se constituye y existe un conocimiento lego empoderado ( paciente experto) pero que al mismo tiempo preserva críticamente la autoridad y práctica médica. 


\subsection{El empoderamiento social del agente lego socava estado y autoridad de la profesión médica}

En esta situación los autores ejemplifican a partir de hallazgos empíricos de Michael Hardey (HARDEY, 2001) y Patricia Drentea y Jennifer L. Moren - Cross (DRENTEA; MOREN-CROSS, 2005) donde colectivos médicos han accionado deliberadamente en monopolizar el conocimiento técnico a efectos de controlar la relación con sus pacientes sobre todo en estrategias de combatir el uso de medicinas alternativas o en el caso de comunidades virtuales dedicadas al tema de la maternidad. En este caso los autores (DENTREA Y OTROS, 2005) y en relación a un sitio web analizado sostienen: "tiene un papel en la desprofesionalización de la medicina y en la consolidación y refuerzo del poder de los movimientos de autoayuda. Este sitio web transfirió la información de ciencia y profesionales, para las mujeres mismas" (DENTREA Y OTROS,2005, p.938).

\subsection{El empoderamiento social del agente lego como generador del fortalecimiento de la racionalidad biomédica.}

En este punto es dónde se verifica la mayor cantidad de consultas bibliográficas, se advierte que el aumento del conocimiento del paciente sobre su salud o enfermedad no implica directamente la pérdida de la autoridad del médico, en todo caso supone cierta barrera a un modo paternalista de atención médica pero no cuestiona la prevalencia del saber profesional. De todas formas, se admite que el empoderamiento social a través de un nuevo bagaje informacional en salud por parte de los agentes legos habrá de reformular el modelo tradicional de relación médico-paciente. En el artículo es abundante la bibliografía referida a que el empoderamiento social en salud a partir de mayor acceso y uso a la información digital no genera cuestionamientos basales al conocimiento técnico experto. Una investigación sobre mujeres y toma de decisiones en salud detecta que ellas confían más en el médico que en su propia experiencia y los autores sugieren que la relación médico - paciente debería fundarse en un intercambio de información y toma de decisiones compartida (HENWOOD, WYATT.S , HART .A, SMITH. J, 2003). En otro estudio (PANDEY; HART; TIWARY, 2003) se afirma que los médicos siguen siendo la principal fuente de información para las mujeres - en el Estado de New Jersey- cuando ellas se orientan a la búsqueda de información en salud. Por otro lado, se investigó en 
grupos de jóvenes respecto a sus necesidades en salud y las estrategias para el acceso a información en condiciones de privacidad (SKINNER; POLAND; BISCOPE, 2003): En este caso internet aparece como una cuestión complementaria siendo la más relevante la que se obtiene a través de los profesionales, en igual sentido arroja evidencia empírica una investigación realizada con adolescentes norteamericanos y británicos (GRAY et al., 2005) donde los profesionales son apreciados como informantes confiables y que parece atendible complementar con información de internet. También hay investigaciones respecto a que la figura del médico sigue siendo la primera fuente de información (COTTEN; GUPTA, 2004). Otros académicos sostienen que el uso de Internet para la búsqueda anónima de información sobre salud y enfermedad aumenta la demanda de atención médica, ello surge de una investigación con personas afectadas por enfermedades consideradas estigmatizantes, tales como: depresión, ansiedad, incontinencia urinaria o herpes (BERGER; WAGNER; BAKER, 2005). Las conclusiones del estudio referido van en la dirección que las consultas de internet sobre salud parecen reforzar el modelo biomédico dominante. Otro elemento significativo refiere a ciertos sitios de información sobre salud en internet donde la que está disponible revela intereses de la industria farmacéutica e institutos médicos (SEALE, 2005). Por último, una investigación realizada en Inglaterra (NETTLETON; BURROWS; O’MALLEY, 2005) donde se concluye que la búsqueda de información de salud en internet refuerza los discursos asentados en la racionalidad biomédica.

\subsection{El conocimiento lego empoderado (paciente experto) y visión crítica de la autoridad y práctica médica}

Así como existen autores que prevén la desprofesionalización de la práctica médica de acuerdo a las "predicciones" de Haug, hay quienes admiten que la existencia del paciente experto habrá de modificar el patrón tradicional de la relación médicopaciente. Más allá de esta dualidad hay quienes tienen visiones donde ambos fenómenos coexisten. A partir del análisis de un foro en internet sobre obesidad y el uso de un fármaco un grupo de investigadores (FOX; WARD; O'ROURKE, 2005a) afirma que la comunicación entre paciente y profesional, convierte a los primeros en colaboradores del segundo a través de su participación activa en el control de su salud y esta situación puede devenir en que ese paciente experto pueda estar aún más sujeto al poder biomédico: "En este contexto, ser un paciente experto no es solo estar informado y comprometido, sino 
estar abierto al escrutinio, la normalización y la estandarización impuestas por el médico" (FOX; WARD; O'ROURKE, 2005a, p. 1299). El mismo grupo de investigadores realizó un segundo estudio (FOX; WARD; O'ROURKE, 2005b): una comunidad virtual en relación a la anorexia donde reivindica el "no tratamiento" siendo la misma una experiencia que se opone a los modelos médicos y sociales dominantes. En investigaciones con tópicos similares (ZIEBLAND, 2004) se considera que:

\begin{abstract}
el hecho de que el paciente tenga acceso a información previamente reservada para los médicos puede, de hecho, implicar una reducción de su autoridad, constituir una amenaza para el control del médico sobre la relación y, por lo tanto, contribuir a la desprofesionalización de la medicina. Sin embargo, se pregunta si Internet continuará siendo utilizada activamente en el futuro y si los profesionales médicos terminarán transformándola en un "apoyo para su trabajo y para su comunicación con los pacientes (ZIEBLAND, 2004, p.1792), preservando así la asimetría de la información y, en consecuencia, el poder en la relación con su paciente (GARBIN; PEREIRA NETO; GUILAM, 2008, p.585).
\end{abstract}

\title{
4.5 La incorporación normativa de la figura del paciente experto en un sistema de
} salud

Tal como se fundamentó en el artículo "Del conocimiento lego al conocimiento experto. Desafíos de la investigación cualitativa y la participación social en salud" (LEVIN, 2019.) la reflexión teórica desde la cual abordamos las investigaciones referidas en numerales 2 y 3 reposan en el conocido enfoque de Anthony Giddens sobre la dialéctica del conocimiento experto -conocimiento lego y la reflexividad (GIDDENS, 1994). Resulta interesante, ante la opción teórica elegida confrontar la perspectiva de Ian Greener cuando - a partir del artículo Expert Patients and Human Agency: Long-term Conditions and Giddens' Structuration Theory, (GREENER, 2008) - examina críticamente el enfoque del Gobierno del Reino Unido para la enfermedad a largo plazo en el capítulo correspondiente al "Paciente experto" del Libro Blanco del Sistema de Salud británico del año 1999. Allí Ian Greener cuestiona teóricamente el enfoque asentado respecto al conocimiento experto y su eventual importancia en el funcionamiento del sistema de salud.

Entre 1999 y 2001, el gobierno trató de hacer hincapié en el desarrollo de la capacidad de autogestión en el área de la enfermedad a largo plazo mediante el uso de una nueva idea, un nuevo modelo de atención: El 'Paciente experto' se presentó en el Libro Blanco de 1999 'Nuestra nación más saludable' debido a que se había considerado que las estrategias de atención anteriores no habían reconocido la forma cómo las personas pueden desempeñar un papel positivo en la construcción de vidas saludables para sí mismas y en contribuir a la salud de otros miembros de la sociedad '( Departamento de Salud, 1999, Sección 3.2). Este enfoque se elaboró aún más mediante la publicación de 'El paciente experto: un nuevo enfoque para el manejo de enfermedades crónicas para el 
siglo XXI' dos años después (Departamento de Salud, 2001b) y se ha convertido, sin mucha estridencia, en una parte central de la dinámica del nuevo mercado de atención en el ServicioNacional de Salud (NHS) que se basa activamente en la introducción generalizada del modelo de atención de pacientes expertos en el sistema de salud (GREENER, 2008, p.273).

La precedente afirmación de Greener toma un primer indicio narrativo en una cita textual de Liam Donaldson (médico británico. Director Médico del NSH Inglaterra entre 1998 y 2010) en: Introducción al PACIENTE EXPERTO:

\begin{abstract}
Este será un programa ambicioso y desafiante para el NHS. Desde hace tiempo se reconoce que las personas con enfermedades crónicas tienen un considerable conocimiento y experiencia de su propia enfermedad. La investigación ha demostrado que, con una capacitación adecuada, esto se puede convertir en habilidades prácticas para permitir que el paciente desempeñe un papel más importante en el manejo de su propia condición. El nuevo programa de pacientes expertos ayudará a crear una nueva generación de pacientes que estén capacitados para actuar con el profesional de la salud que los atiende para reducir el dolor, mejorar el uso de medicamentos y mejorar su calidad de vida en general. Los pacientes recibirán el apoyo para ayudarlos a tomar más control de su propia salud y tratamiento, hacer un uso más apropiado de los servicios sociales y de salud y sentirse empoderados en su relación con los profesionales de la salud. Profesor Liam Donaldson, Director Médico, 14 de septiembre de 2001 (GREENER, 2008, p.274).
\end{abstract}

Greener adopta una postura crítica ante la incorporación de la figura del "Paciente Experto" en el sistema de salud británico pero su crítica está fundada no en los propósitos que animan dicha incorporación (noción de autocuidado y compromiso individual con un adecuado estado de salud) sino en la falta de evidencia científica que justifique su implantación institucional:

El 'paciente experto' no es solo un enfoque que desafía las preconcepciones sobre la naturaleza del conocimiento y el poder médicos, suponiendo que los pacientes pueden autocontrolar sus condiciones en mayor medida que en la actualidad. También sugiere que el conocimiento médico puede ser apropiado por pacientes con afecciones a largo plazo y complementado por el conocimiento laico adquirido de los grupos de autoayuda (GREENER, 2008, p.275).

Otros autores complementan su visión:

Cuando las personas adquieren afecciones a largo plazo, como asma o EM, desarrollan experiencia en el manejo de su afección, aprenden a reducir los peores efectos de la enfermedad y también a "adaptar" su propio paquete de atención y uso de drogas. Como tal, se convierten en expertos en el manejo de su propia condición, una experiencia a menudo basada no en el aprendizaje de libros, sino en su propio cuidado diario (WILSON, 2001, p134).

El aumento a nivel mundial de las enfermedades crónicas ha dado lugar a un énfasis creciente en las iniciativas de autocuidado. En el caso del Reino Unido esto se ejemplifica en la inclusión del Programa de Pacientes Expertos. En este punto es donde Greener apunta al cerno teórico de dicha política y se pregunta en qué medida ésta desafía 
las relaciones de poder convencionales entre médicos y pacientes. No se trata de una simple traslación teórica de lo que Giddens denomina conocimiento experto y conocimiento lego. Greener sostiene que este último difícilmente pueda situarse en un lugar de competencia profesional equiparable al de los médicos. En particular, se debate la definición de experto y si una comprensión profesional del término se puede conciliar con la experiencia del paciente (GREENER, 2008).

Un funcionamiento eficaz del programa Paciente Experto supone la existencia no solo de un sujeto reflexivo, proactivo y en condiciones objetivas de auto validez sino con la capacidad de asumir responsabilidades emergentes de su propia atención. Por otra parte, deben generarse los dispositivos del sistema de salud articulados con la propia demanda que el paciente experto- en esta nueva condición- habrá de generar. Ello supone un cambio radical de la cultura médica (TATTERSAL, 2002).

Greener aporta otros enfoques analíticos. Por un lado, la desprofesionalización médica que puede suponer el programa de Paciente Experto y una consecuente política regresiva en materia de asignación de recursos estatales a programas de cuidado. Una cuestión agregada radica en que la figura del Paciente Experto supone per se - ante la simple invitación a participar en el autocuidado de la salud- la condición necesaria para el empoderamiento social de los enfermos y ello no es una consecuencia inmediata e ineluctable (PATERSON, 2001)

Y advierte ciertos riesgos:

Es necesario lograr un equilibrio entre el cuidado y el empoderamiento: si solo se considera el primero, existe el peligro de imponer un discurso de disciplina foucaultiano a los pacientes expertos, donde consideran que cualquier dependencia de la profesión médica es un fracaso. Los pacientes, con la experiencia y el conocimiento que han adquirido sobre sus afecciones, claramente pueden hacer una contribución positiva hacia su propia atención, pero esto debe atenuarse con un reconocimiento de la dependencia que ponemos en los médicos: dependencia en un sentido positivo y afectuoso. Si el estado se refiere continuamente a los ahorros de recursos que podrían lograrse mediante un mayor uso de los programas de pacientes expertos, esto pone a la profesión médica bajo una presión considerable para introducir el programa donde sea inapropiado (GREENER, 2008, p.287).

Un señalamiento sustantivo radica, para los pacientes crónicos en particular, que el manejo de una afección que suponga uso de medicamentos en situaciones imprecisas puede aumentar el número de consultas médicas y debe tomarse en cuenta que algunos pacientes con discapacidades físicas o psicológicas no suelen estar en la mejor situación para negociar su atención (GREENER, 2008). 


\subsection{Aportes a la discusión}

Los estudios identificados en los dos artículos revisados: A internet, o paciente expert e a prática médica: uma análise bibliográfica (GARBIN; PEREIRA NETO; GUILAM, 2008) y Expert Patients and Human Agency:Long-term Conditions and Giddens' Structuration Theory (GREENER, 2008) convergen en nuestro tópico de trabajo en lo relativo a la circulación del conocimiento lego y el experto y sus eventuales implicancias para la investigación cualitativa como enfoque científico metodológico y a la participación social como estrategia de investigación en el campo de la salud pública. En tal sentido es importante señalar algunos aspectos convergentes con nuestra preocupación teórica y metodológica y que los autores revisados observan como relevantes.

\subsubsection{Sistematización del artículo "A internet, o paciente expert e a prática médica:} uma análise bibliográfica” de Helena Beatriz da Rocha Garbin; André de Faria Pereira Neto y María Cristina Rodrigues Guilam, (GARBIN; PEREIRA NETO; GUILAM, 2008)

El papel de internet en el campo de la información en salud se ha vuelto central en la vida cotidiana, no solo en lo relacionado a la obtención de información técnica y científica sino también en la conformación de comunidades virtuales y grupos de apoyo para determinados tipos de enfermedades o afecciones a la salud. La emergencia y consolidación de comunidades virtuales en apoyo a grupos de pacientes se ve acompañado de otro epifenómeno y es la existencia de muchos sitios web que difunden información contradictoria o errónea.

Es incuestionable el potencial impacto del caudal informacional de internet sobre la relación médico - paciente en la medida que puede elevar el poder de decisión del paciente y ello, a su vez, puede significar un cuestionamiento indudable de la capacitación y la autoridad profesional médica. Asociado al punto anterior, el aumento del saber en el actor lego y el desafío que ello supone para la formación permanente del actor profesional crea un ambiente favorable para una toma de decisiones más compartida entre ambos actores.

Un aporte sustantivo lo constituye la vigorización del debate sobre la desprofesionalización de la práctica médica a partir de los enfoques de Haug y Friedson 
siendo dicho aspecto una dimensión a incorporar en la dialéctica del conocimiento lego y experto en salud.

Un señalamiento relevante es la situación paradojal- a partir de la contrastación empírica- respecto a la indudable interferencia de internet en la relación médico-paciente y dónde, a pesar de la abundante información en relación al tópico salud, los médicos han fortalecido posiciones de poder y existen tecnologías sólidas y dominantes que resultan difícilmente "apropiables" por parte de los no médicos.

Por último, el artículo propone una mirada de extrema sensatez en relación a que los ciudadanos históricamente, cuando asumen el rol de pacientes siempre buscan información fuera del circuito médico, así como indagan en otras experiencias sobre determinada enfermedad, instituciones o profesionales de la salud. Siempre ha existido dicha trayectoria terapéutica, al día de hoy allanada en gran forma por la existencia de internet

\subsubsection{Sistematización del artículo "Expert Patients and Human Agency: Long-term Conditions and Giddens' Structuration Theory" de Ian Greener (GREENER, 2008)}

El cuestionamiento teórico al Programa Paciente Experto y su falta de evidencia empírica para justificar incorporación al sistema de salud inglés aparece- en el marco de este artículo- como una buena señal de alarma epistemológica para quienes estamos construyendo marcos de interpretación de la acción social en salud a partir de la relación paciente y médico y sobre todo a partir de la metáfora sociológica de Giddens sobre la circulación de los saberes legos y expertos en tiempos de modernidad.

Un tema relevante es señalado (al igual que en el artículo anterior) en relación al "empoderamiento social" de los actores legos y la eventual amenaza que ello implica sobre la desprofesionalización de la medicina a la vez de apuntar a un proceso convergente de restricción de fondos estatales al cuidado en salud de las personas.

Por último, debe señalarse un atributo significativo: Refiere a los procesos de empoderamiento social de actores tradicionalmente subordinados (paciente $\mathrm{o}$ conocimiento lego) que está implícito en las estrategias de auto cuidado. Dicho fenómeno aparece en clave de individuación (auto cuidado, saber individual) lo cual también funciona como alerta epistemológica respecto a las estrategias de participación social en salud que hemos aludido en el presente artículo. 


\section{Conclusiones}

En este punto retomamos las preguntas que iniciaron el presente artículo:

- ¿Existen indicadores de empoderamiento por parte de actores socialmente subordinados en el sistema de salud? En el caso del Insumo 1 se construyeron categorías analíticas robustas: La carrera del paciente; Conocimiento tipo 1 y Conocimiento tipo 2. Si observamos Insumo 2, ante la misma pregunta se tienen dimensiones explicativas del proceso de interacción social en el que participaron los agentes legos: Autoevaluación del conocimiento adquirido; Rol de los asesores; Rol como integrante del panel ciudadano; Evaluación de la audiencia pública y Recomendaciones de los ciudadanos. En ambas investigaciones se detectan procesos reflexivos orientados a la adquisición de nuevos conocimientos por parte de los agentes legos significando ello cambios en las posiciones del campo social entre el saber lego y el experto. Es un dato relevante para comprender la acción de los sujetos (expertos y legos) en el área de la Salud y admite ser evaluado como insumo trascendente a la hora de diseñar políticas públicas en el sector. Por último, la revisión bibliográfica realizada (Insumo 3). Explícita o implícitamente el insumo 3 (revisión de artículos ) develó aspectos comunes a las investigaciones precedentes: a: el acceso a información masiva en salud da cuenta de procesos cognitivos por parte de actores legos que frecuentemente pone a prueba la capacitación y la autoridad profesional de los médicos; b: parece haber consensos que los citados procesos cognitivos en actores legos alientan procesos de tomas de decisión mas compartidas entre pacientes y médicos; c: también parece existir acuerdo en un número importante de autores que el empoderamiento social de los actores legos puede ser un catalizador de prácticas desprofesionalizantes de la práctica médica; d: Algunas prácticas desprofesionalizantes parecen solapar restricciones presupuestales del Estado al sistema de cuidados en salud (Programa de Paciente Experto en Gran Bretaña) e: Las estrategias de promoción del auto cuidado y su impronta individualizadora parecen generar prácticas de empoderamiento social diferentes a lo que suele denominarse participación social en salud (enfoque de salud colectiva).

- ¿Cómo se expresa la interacción social en los diferentes procesos de decisión?:Todo indica, en ambas investigaciones (Insumos 1 y 2 ) la asunción de un rol activo por parte de los agentes legos (enfermos renales y panelistas del Juicio Ciudadano) donde existen innumerables indicadores del "empoderamiento" de aquellos que -en términos de interacción social- están en una posición subordinada al saber experto. En 
este punto es interesante cotejar los procesos cognitivos por parte de agentes legos en ambas investigaciones y algunas evidencias empíricas de los artículos analizados (Insumo 3). a: existe una peculiar consistencia entre dichos procesos y la conformación de comunidades virtuales y grupos de apoyo para determinados tipos de enfermedades observadas en el artículo de Garbin, Pereira Neto e Guilam; b: La expresión "Carrera del Paciente" (Insumo 1) encuentra en el articulo citado su homología en la expresión “ Trayectoria terapéutica" donde existe una constatación desde el sentido común respecto a que los ciudadanos cuando asumen el rol de pacientes siempre buscan información fuera del circuito médico

- ¿Qué técnicas, desde la investigación cualitativa en salud, se revelan eficaces para construir evidencia empírica sólida respecto a los actores sociales, su trayectoria y posición en el sistema de salud? En el caso del Insumo 1, un primer elemento significativo se deriva del trabajo con fuentes secundarias que fueron el sustento empírico de una anterior investigación sobre la ERC respaldada por un exhaustivo trabajo de campo. Un segundo elemento refiere a que dicho corpus discursivo fué muy eficaz para aplicar un modelo teórico basado en la circulación social de saberes diversos (legos y expertos) tal como lo conceptualizó

Anthony Giddens. En el caso del insumo 2, el proceso cognitivo de adquisición de nuevos saberes por parte de los agentes legos también da cuenta de dicha conceptualización. Por último, la investigación cualitativa como estrategia y la investigación participativa como técnica se revelan eficaces en su pretensión ontológica. El desafío radica en persuadir a la institucionalidad política de asumir a la investigación social aplicada como insumo esencial en la construcción de políticas públicas.

- ¿Son eficaces las categorías analíticas de conocimiento lego y conocimiento experto en salud para comprender los diversos procesos de interacción social en el campo de la salud? Cuando utilizamos las expresiones "conocimiento lego" y "conocimiento experto" asociadas al concepto de reflexividad aludimos directamente a Anthony Giddens (GIDDENS, 1994). Dicho autor define la reflexividad como una cualidad específica de las prácticas sociales en la modernidad. Es indisociable de la recursividad, entendida esta como la reproducción ordenada de las prácticas sociales. Ambas constituyen el punto de intersección en las diferentes capacidades de los agentes: Tener noción de saber lo que hacen y porqué lo hacen, es decir, tener la capacidad de monitorear sus acciones. Esto a su vez, le permite interacción con otros agentes generando a su vez nuevas prácticas sociales autorreproducidas reflexivamente. Supone una relación 
dialéctica entre el saber profano y el experto. En esa relación habitan la generación e intercambio de saberes donde el conocimiento lego- desde la reflexividad del agenteincorpora un nuevo conocimiento que a su vez ingresa en el espacio de la disputa social, normalmente bajo la forma del conflicto y el acuerdo. La reflexividad, en tanto concepto teórico interpretado bajo el lente de Anthony Giddens habilita a comprender la circulación social de nuevos conocimientos por parte de los agentes legos (portadores de ERC y familiares, por ejemplo, Insumo 1) desarrollando nuevos y recursivos procesos de interacción social con el conocimiento experto. Ello se expresa, en la tríada conceptual presentada: Carrera del paciente, conocimiento Tipo 1 y conocimiento Tipo 2. En este punto debemos señalar (y a beneficio de inventario de futuras reflexiones al respecto) que en el artículo de Ian Greener interviene directamente sobre esta formulación teórica de Giddens en forma muy crítica pero haciendo hincapié en que la incorporación del Programa del Paciente Experto en el sistema de salud inglés obedece a un traslado mecánico y general de la construcción teórica sociológica a un esquema de gestión particularizado de atención a la salud.

Obviamente, en este artículo hacemos una primera aproximación a esta situación de conflicto entre la teoría social y la vida cotidiana en un sistema de salud a partir de los autores reseñados.

Agradecimiento: A la memoria del Profesor Francisco Mercado (fallecido en Guadalajara, México el día 6 de enero de 2019). Gracias por el impulse.

\section{Referencias}

BERGER, M.; WAGNER, T.H.; BAKER, L.C. Internet use and stigmatized illness. Social Science \& Medicine, [S.I.], v.61, n.8, p.1821, oct. 2005.

COTTEN, S.R.; GUPTA, S.S. Characteristics of online and offline health information seekers and factors that discriminate between them. Social Science \& Medicine, [S.I.], v.59, n.9, p.1795-806, nov. 2004.

GARBIN, H.B.R.; PEREIRA NETO, A.F.; GUILAM, M.C.R. A internet, o paciente expert e a prática médica: uma análise bibliográfica. Interface Comunicação Saúde Educação, San Pablo.Brasil, v.12, n.26, p.579-88, jul./set. 2008.

DRENTEA, P.; MOREN-CROSS, J.L. Social capital and social support on the web: the case of an internet mother site. Sociology of Health \& Illness, [S.I.], v.27, n.7, p.920-943, nov. 2005.

FOX, N.J.; WARD, K.; O'ROURKE, A. The 'expert patient': empowerment or medical dominance? The case of weight loss, pharmaceutical drugs and the internet. Social Science \& Medicine, [S.I.], v.60, n.6, p.1299-1309, mar. 2005a. 
FOX, N.J.; WARD, K.; O'ROURKE, A. Pro-anorexia, weight-loss drugs and the internet: an 'anti-recovery' explanatory model of anorexia. Social Science \& Medicine, [S.I.], v.27, n.7, p.944-971, Marzo 2005b.

FUENTES, G. El sistema de salud uruguayo en la post dictadura: análisis de la reforma del Frente Amplio y las condiciones que la hicieron posible en Revista Uruguaya de Ciencia Política, Montevideo, v.19, n.1, ene. 2010.

GIDDENS, A. La índole reflexiva de la modernidad. En: GIDDENS, A. Consecuencias de la Modernidad. Madrid: Alianza Editorial, 1994. p.44-52.

GOFFMAN, E. Cap. La carrera moral del paciente mental. En: GOFFMAN, E. Internados. Ensayos sobre la situación social de los enfermos mentales. Buenos Aires: Amorrortu editors, 1972. p. 132.-172.

KLEIN. JD, NOYCE. PR, SESSELBERG. TS, CANTRILL. JA .Health information-seeking behaviour in adolescence: the place of the internet. Social Science \& Medicine, [S.I.], v.60, n.7, p.1467-1478, abr. 2005.

GREENER, I. Expert Patients and Human Agency: Long-term Conditions and Giddens' Structuration Theory. Social Theory \& Health, [S.I.], v. 6, n.4, p 273-290, set. 2008.

HARDEY, M. Doctor in the house: the internet as a source of lay health knowledge and the challenge to expertise. Sociology of Health \& Illness, [S.I.], v.21, n.6, p.820-823, dic. 2001.

HENWOOD. F.; WYATT. S.; HART, A., SMITH. J. Ignorance is a bliss sometimes: constraints on the emergence of the "informed patient" in the changing landscape of health information. Sociology of Health \& Illness, [S.I.], v.25, n.6, p.589-607, ago. 2003.

LÁZARO, M. et al. Juicios Ciudadanos en Uruguay: dos experiencias de participación pública deliberativa en ciencia y tecnología. Proyecto Regional de Diálogo Democrático del Centro Regional para América Latina y el Caribe del Programa de las Naciones Unidas para el Desarrollo (PNUD): Montevideo Uruguay. 2013.

LEVIN R. Del conocimiento lego al conocimiento experto. Desafíos de la investigación cualitativa y la participación social en salud Congreso Iberoamericano de Investigación Cualitativa en Salud. In: CONGRESSO IBERO-AMERICANO DE INVESTIGAÇÃO QUALITATIVA, 8., 2019. Anais... Aveiro: Ludomedia, 2019. p. 549-558.

Ley 18.211,2008. Creación del Sistema Nacional Integrado de Salud. https://www.ilo.org/dyn/natlex/docs/ELECTRONIC/86022/96727/F565346650/URY86. Ley 18.211. Poder Legislativo. Publicada D.O. 13 dic/007 - No 27384. Diciembre 2007

MERCADO, F., LEVIN R. La atención a la enfermedad renal en Uruguay: la perspectiva de individuos con trasplante renal. Cadernos de Saúde Pública, Rio de Janeiro, v. 33, n. 10, p. 112, out. 2017

NETTLETON, S.; BURROWS, R.; O'MALLEY, L. The mundane realities of the everyday lay use of the internet for health, and their consequences for media convergence. Sociology of Health \& Illness, [S.I.], v.27, n.7, p. 972-992, nov. 2005.

PANDEY, S.K.; HART, J.J.; TIWARY, S. Women's health and the internet: understanding emerging trends and implications. Social Science \& Medicine, [S.I.], v.56, n.1, p.179-191, jan. 2003. 
PATERSON, B. The myth of empowerment in chronic illness. Journal of Advanced Nursing, [S.I.], v. 34, n. 5, p. 575-581, dez. 2001.

SEALE, C. New directions for critical internet health studies: representing cancer experience on the web. Sociology of Health \& Illness, [S.I.], n.4, p.515-40, jul. 2005.

SKINNER, H.; BISCOPE, S.; POLAND, B. Quality of internet access: barrier behind internet use statistics. Social Science $\boldsymbol{\&}$ Medicine, [S.I.], v.57, n.5, p.875-80, set. 2003.

TATTERSALL R. The expert patient: a new approach to chronic disease management for the twenty-first century. Clinical Medicine, [S.I.], v. 2, p. 227-229, maio. 2002.

WILSON P. A policy analysis of the Expert Patient in the United Kingdom: self-care as an expression of pastoral power. Health and Social Care in the Community, [S.I.], v. 9, p. 134142, dez. 2001.

ZIEBLAND, S. The importance of being expert: the quest for cancer information on the internet. Social Science \& Medicine, [S.I.], v.59, n.9, p.1783-1793, nov. 2004.

Recebido em: 05 de fevereiro de 2020.

Aceito em: 04 de março de 2020. 